Kragujevac Journal of Mathematics

Volume 46(1) (2022), Pages 39-47.

\title{
REFINING SOME INEQUALITIES FOR FRAMES WITH SPECHT'S RATIO
}

\section{FAHIMEH SULTANZADEH ${ }^{1}$, MAHMOUD HASSANI ${ }^{1}$, MOHSEN ERFANIAN OMIDVAR ${ }^{1}$, AND RAJAB ALI KAMYABI GOL ${ }^{2}$}

\begin{abstract}
We give a new lower bound in some inequalities for frames in a Hilbert space. If $\left\{f_{i}\right\}_{i \in I}$ is a Parseval frame for the Hilbert space $\mathbb{H}$ with frame operator $S f=\sum_{i \in I}\left\langle f, f_{i}\right\rangle f_{i}$, then, for every $J \subset I$ and $f \in \mathbb{H}$, we have

$$
\left(\frac{1+2 \alpha}{2+2 \alpha}\right)\|f\|^{2} \leq \sum_{i \in J}\left|\left\langle f, f_{i}\right\rangle\right|^{2}+\left\|\sum_{i \in J^{c}}\left\langle f, f_{i}\right\rangle f_{i}\right\|^{2},
$$

where $\alpha=\inf \left\{R\left(\frac{\left\|S_{J^{c}} f\right\|}{\left\|S_{J} f\right\|}\right): f \in \mathbb{H}, J \subset I\right\}$ with Specht's ratio $R$. Also we obtain some improvements of the inequalities for general frames and alternate dual frames under suitable conditions. Our results refine the remarkable results obtained by Balan et al. and Gavruta.
\end{abstract}

\section{INTRODUCTION}

Frame theory was introduced by Duffin and Schaeffer [6] in 1952. Frames are an essential tool for many emerging applications. Their main advantage is the fact that frames can be designed to be redundant while still providing reconstruction formulas. Due to their numerical stability, tight frames and, in particular, Parseval frames are of increasing interest in applications (see $[5,7,13])$. Let $(\mathbb{H},\langle\cdot, \cdot\rangle)$ be a separable Hilbert space. We denote by $L(\mathbb{H})$ the algebra of all linear operators on $\mathbb{H}$. The space $\ell^{2}(I)$ is the set of $\left\{a_{i}\right\}_{i \in I}$ such that $a_{i} \in \mathbb{C}$ and $\sum_{i \in I}\left|a_{i}\right|^{2}<\infty$ when $I$ is a finite or countable set. A frame for $\mathbb{H}$ is a family of vectors $F=\left\{f_{i}\right\}_{i \in I}$ in $\mathbb{H}$ which satisfies

$$
A\|f\|^{2} \leq \sum_{i \in I}\left|\left\langle f, f_{i}\right\rangle\right|^{2} \leq B\|f\|^{2}, \quad \text { for every } f \in \mathbb{H},
$$

Key words and phrases. Specht's ratio, frame, Parseval frame, inequality. 2010 Mathematics Subject Classification. Primary: 42C15.

DOI 10.46793/KgJMat2201.039S

Received: May 18, 2019.

Accepted: August 16, 2019. 
for positive constants $0<A \leq B$. The bounded, invertible, and positive linear operator $S: \mathbb{H} \rightarrow \mathbb{H}$ defined by

$$
S f=\sum_{i \in I}\left\langle f, f_{i}\right\rangle f_{i}, \quad f \in \mathbb{H}
$$

is known as the frame operator associated to $F$. It allows reconstruction of each vector $f \in \mathbb{H}$ in terms of the family $F$ as follows:

$$
f=\sum_{i \in I}\left\langle f, S^{-1} f_{i}\right\rangle f_{i}=\sum_{i \in I}\left\langle f, f_{i}\right\rangle S^{-1} f_{i} .
$$

If $F$ is a Parseval frame, that is, $S=i d$, then the reconstruction formula resembles the Fourier series of $f$ associated to an orthonormal basis $B=\left\{b_{j}\right\}_{j \in J}$ of $\mathbb{H}$ :

$$
f=\sum_{j \in J}\left\langle f, b_{j}\right\rangle b_{j}
$$

but the frame coefficients $\left\{\left\langle f, f_{i}\right\rangle\right\}_{i \in I}$ given by $F=\left\{f_{i}\right\}_{i \in I}$ allow us to reconstruct $f$ even when some of these coefficients are corrupted (see [6]).

Balan et al. [1] and Gavruta [9] established several identities and inequalities for frames in Hilbert spaces. Furuichi [8] refined Young inequalities with Specht's ratio and introduced their properties. In this paper, first we use this improved inequality in some inequalities for Parseval frames and get new inequalities. Thereafter we give improvements for general frames. However our main focus will be on Parseval frames because of their importance in applications, particularly for signal processing. Finally we give improvements for alternative dual frames too.

\section{Notation and Preliminary Results}

2.1. Frames in Hilbert space. In the definition of frame for Hilbert spaces, the optimal constants (maximal for $A$ and minimal for $B$ ) are known as the upper and lower frame bounds, respectively. If $A=B$, then this frame is called an $A$-tight frame, and if $A=B=1$, then it is called a Parseval frame. If a family of vectors $F=\left\{f_{i}\right\}_{i \in I}$ satisfies the upper bound condition (1.1), we call $F$ a Bessel family. Associated with each frame $F=\left\{f_{i}\right\}_{i \in I}$, there are three linear and bounded operators:

$T: \ell^{2}(I) \rightarrow \mathbb{H}, T x=\sum_{i \in I}\left\langle x, e_{i}\right\rangle f_{i}$ (synthesis operator);

$T^{*}: \mathbb{H} \rightarrow \ell^{2}(I), T^{*}(f)=\left\{\left\langle f, f_{i}\right\rangle\right\}_{i \in I}$, (analysis operator);

$S: \mathbb{H} \rightarrow \mathbb{H}, S f=T T^{*} f=\sum_{i \in I}\left\langle f, f_{i}\right\rangle f_{i}$ (frame operator),

where $\left\{e_{i}\right\}_{i \in I}$ is the standard orthonormal basis of $\ell^{2}(I)$. The inequalities (1.1) imply that $S$ is a (positive) self-adjoint invertible operator, and it allows reconstruction of each vector $f \in \mathbb{H}$ in terms of the family $F$ as follows:

$$
f=\sum_{i \in I}\left\langle f, S^{-1} f_{i}\right\rangle f_{i}=\sum_{i \in I}\left\langle f, f_{i}\right\rangle S^{-1} f_{i} .
$$

Then the family $\left\{\tilde{f}_{i}\right\}_{i \in I}$, where $\tilde{f}_{i}=S^{-1} f_{i}, i \in I$, is also a frame for $\mathbb{H}$ called the canonical dual frame of the $F=\left\{f_{i}\right\}_{i \in I}$. 
In general, the Bessel family $\left\{g_{i}\right\}_{i \in I}$ is called an alternative dual of the frame $F=\left\{f_{i}\right\}_{i \in I}$ if the following formula holds:

$$
f=\sum_{i \in I}\left\langle f, g_{i}\right\rangle f_{i}, \quad \text { for all } f \in \mathbb{H} .
$$

If $\left\{f_{i}\right\}_{i \in I}$ is a frame for $\mathbb{H}$ for every $J \subset I$ we define the operator

$$
S_{J} f=\sum_{i \in J}\left\langle f, f_{i}\right\rangle f_{i}
$$

and denote $J^{c}=I \backslash J$. It follows that $S=S_{J}+S_{J^{c}}$. By this definition, it is clear that if $J_{1} \subseteq J_{2}$, then $\left\|S_{J_{1}} f\right\| \leq\left\|S_{J_{2}} f\right\|$.

For more details, we refer the reader to $[2-4,10,12]$. In [1], Balan et al. proved the following identity for Parseval frames:

$$
\sum_{i \in J}\left|\left\langle f, f_{i}\right\rangle\right|^{2}-\left\|\sum_{i \in J}\left\langle f, f_{i}\right\rangle f_{i}\right\|^{2}=\sum_{i \in J^{c}}\left|\left\langle f, f_{i}\right\rangle\right|^{2}-\left\|\sum_{i \in J^{c}}\left\langle f, f_{i}\right\rangle f_{i}\right\|^{2} .
$$

Moreover, in [1] the following inequality was obtained

$$
\frac{3}{4}\|f\|^{2} \leq \sum_{i \in J}\left|\left\langle f, f_{i}\right\rangle\right|^{2}+\left\|\sum_{i \in J^{c}}\left\langle f, f_{i}\right\rangle f_{i}\right\|^{2} .
$$

See $[9,11]$ for further details. In fact, identity $(2.1)$ was obtained as a particular case from the following result for general frames:

$$
\sum_{i \in J}\left|\left\langle f, f_{i}\right\rangle\right|^{2}+\sum_{i \in I}\left|\left\langle S_{J^{c}} f, \tilde{f}_{i}\right\rangle\right|^{2}=\sum_{i \in J^{c}}\left|\left\langle f, f_{i}\right\rangle\right|^{2}+\sum_{i \in I}\left|\left\langle S_{J} f, \tilde{f}_{i}\right\rangle\right|^{2} .
$$

Inequality (2.2) leads us to introduce, for a Parseval frame, the numbers

$$
\begin{aligned}
& v_{+}(F ; J)=\sup _{f \neq 0} \frac{\left\|\sum_{i \in J}\left\langle f, f_{i}\right\rangle f_{i}\right\|^{2}+\sum_{i \in J^{c}}\left|\left\langle f, f_{i}\right\rangle\right|^{2}}{\|f\|^{2}}, \\
& v_{-}(F ; J)=\inf _{f \neq 0} \frac{\left\|\sum_{i \in J}\left\langle f, f_{i}\right\rangle f_{i}\right\|^{2}+\sum_{i \in J^{c}}\left|\left\langle f, f_{i}\right\rangle\right|^{2}}{\|f\|^{2}} .
\end{aligned}
$$

Recall that $v_{+}(F ; J)$ is called the upper index of $F$ relative to $J$ and $v_{-}(F ; J)$ is called the lower index of $F$ relative to $J$.

Gavruta [9] presented basic properties of these indexes.

2.2. Improved Young inequality with Specht's ratio. The well-known Young inequality says that $(1-v) a+v b \geq a^{1-v} b^{v}$ for positive numbers $a, b$ and $v \in[0,1]$. A refinement of this inequality is given in the following proposition.

Lemma 2.1 ([8]). Specht's ratio

$$
R(h) \equiv \frac{h^{\frac{1}{h-1}}}{e \log h^{\frac{1}{h-1}}}, \quad h \neq 1, h>0,
$$

has the following properties:

(i) $R(1)=1$ and $R(h)=R(1 / h)>1$ for $h>0$; 
(ii) $R(h)$ is a monotone increasing function on $(1, \infty)$;

(iii) $R(h)$ is a monotone decreasing function on $(0,1)$.

Proposition 2.1 ([8]). For $a, b>0$ and $v \in[0,1]$, it follows that

$$
(1-v) a+v b \geq R\left(\left(\frac{b}{a}\right)^{r}\right) a^{1-v} b^{v}
$$

where $r \equiv \min \{v, 1-v\}$ and $R(\cdot)$ is Specht's ratio.

2.3. Operator Theory. We first state a basic result from operator theory. The following lemma appeared in [1].

Lemma 2.2. If $S, T \in L(\mathbb{H})$ satisfy $S+T=i d$, then $S-T=S^{2}-T^{2}$.

Proof. The proof follows from

$S-T=S-(i d-S)=2 S-i d=S^{2}-\left(i d-2 S+S^{2}\right)=S^{2}-(i d-S)^{2}=S^{2}-T^{2}$.

\section{Main Results}

In this section, first we improve the left-hand-side of inequality (2.2) with Specht's ratio. Thereafter in Lemma 3.1 improvements for self-adjoint operators are given, which we apply for general frames. Finally in Theorems 3.3 and 3.4, we present the results for alternate dual frames.

Theorem 3.1. If $\left\{f_{i}\right\}_{i \in I}$ is a Parseval frame for the Hilbert space $\mathbb{H}$ with frame operator $S$, then, for every $\emptyset \neq J \subset I$ and $f \in \mathbb{H}$, it follows that

$$
\left(\frac{1+2 \alpha}{2+2 \alpha}\right)\|f\|^{2} \leq \sum_{i \in J}\left|\left\langle f, f_{i}\right\rangle\right|^{2}+\left\|\sum_{i \in J^{c}}\left\langle f, f_{i}\right\rangle f_{i}\right\|^{2},
$$

where $\alpha=\inf \left\{R\left(\frac{\left\|S_{J} f\right\|}{\left\|S_{J} f\right\|}\right): f \in \mathbb{H}, J \subset I\right\}$.

Proof. Since

$$
\|f\|^{2}=\left\|S_{J} f+S_{J^{c}} f\right\|^{2} \leq\left\|S_{J} f\right\|^{2}+\left\|S_{J^{c}} f\right\|^{2}+2\left\|S_{J} f\right\|\left\|S_{J^{c}} f\right\|,
$$

by letting $v=\frac{1}{2}, a=\left\|S_{J} f\right\|$ and $b=\left\|S_{J^{c}} f\right\|$ in Proposition 2.1, we have

$$
\|f\|^{2} \leq\left(\left\|S_{J} f\right\|^{2}+\left\|S_{J^{c}} f\right\|^{2}\right)\left(1+\frac{1}{R\left(\frac{\left\|S_{J^{c}} f\right\|}{\left\|S_{J} f\right\|}\right)}\right) .
$$

Put $\alpha=\inf \left\{R\left(\frac{\left\|S_{J} f\right\|}{\left\|S_{J} f\right\|}\right): f \in \mathbb{H}, J \subset I\right\}$, then

$$
\left\langle\left(\frac{\alpha}{1+\alpha}\right) i d f, f\right\rangle \leq\left\langle\left(S_{J}^{2}+S_{J^{c}}^{2}\right) f, f\right\rangle .
$$

This implies that

$$
\left(\frac{\alpha}{1+\alpha}\right) i d \leq S_{J}^{2}+S_{J^{c}}^{2}
$$


So,

$$
\left(\frac{\alpha}{1+\alpha}+1\right) i d \leq S_{J}+S_{J^{c}}^{2}+S_{J^{c}}+S_{J}^{2}
$$

Now, by applying Lemma 2.2, it follows that

$$
\left(\frac{1+2 \alpha}{2+2 \alpha}\right) i d \leq S_{J}+S_{J^{c}}^{2}
$$

Hence,

$$
\left(\frac{1+2 \alpha}{2+2 \alpha}\right)\|f\|^{2} \leq\left\langle S_{J} f, f\right\rangle+\left\langle S_{J^{c}} f, S_{J^{c}} f\right\rangle=\sum_{i \in J}\left|\left\langle f, f_{i}\right\rangle\right|^{2}+\left\|\sum_{i \in J^{c}}\left\langle f, f_{i}\right\rangle f_{i}\right\|^{2} .
$$

Note that for $\alpha=1$, inequality (3.1) is the same as inequality (2.2) and that for $\alpha>1,(3.1)$ is an improvement of (2.2).

Corollary 3.1. Let $F=\left\{f_{i}\right\}_{i \in I}$ be a Parseval frame and let $J \subset I$. Then

$$
\frac{1+2 \alpha}{2+2 \alpha} \leq v_{-}(F ; J) \leq v_{+}(F ; J) \leq 1,
$$

where $\alpha=\inf \left\{R\left(\frac{\left\|S_{J^{c}} f\right\|}{\left\|S_{J} f\right\|}\right): f \in \mathbb{H}, J \subset I\right\}$.

Proof. By using Theorem 3.1 and the hypothesis that $F$ is a Parseval frame, we have

$$
\left(\frac{1+2 \alpha}{2+2 \alpha}\right)\|f\|^{2} \leq \sum_{i \in J}\left|\left\langle f, f_{i}\right\rangle\right|^{2}+\left\|\sum_{i \in J^{c}}\left\langle f, f_{i}\right\rangle f_{i}\right\|^{2} \leq\|f\|^{2}
$$

So,

$$
\frac{1+2 \alpha}{2+2 \alpha} \leq \frac{\sum_{i \in J}\left|\left\langle f, f_{i}\right\rangle\right|^{2}+\left\|\sum_{i \in J^{c}}\left\langle f, f_{i}\right\rangle f_{i}\right\|^{2}}{\|f\|^{2}} \leq 1
$$

Hence,

$$
\frac{1+2 \alpha}{2+2 \alpha} \leq v_{-}(F ; J) \leq v_{+}(F ; J) \leq 1
$$

In the following lemma, we give an improvement of the inequality proved in [9, Theorem 2.1], under some conditions. Then we present Theorem 3.2 for general frames.

Lemma 3.1. Let $T_{1}, T_{2} \in L(\mathbb{H})$ be self-adjoint operators satisfying $T_{1}+T_{2}=i d$, such that $T_{1} \geq \frac{k}{k+1} i d$, where $k \in \mathbb{N}$ and $k>3$. Then

$$
\frac{k}{k+1}\|f\|^{2} \leq\left\langle T_{1} f, f\right\rangle+\left\|T_{2} f\right\|^{2}=\left\langle T_{2} f, f\right\rangle+\left\|T_{1} f\right\|^{2}, \quad \text { for } f \in \mathbb{H} .
$$


Proof. From our assumptions, we have

$$
\begin{aligned}
\left\langle T_{2} f, f\right\rangle+\left\|T_{1} f\right\|^{2} & =\left\langle\left(i d-T_{1}\right) f, f\right\rangle+\left\langle T_{1}^{2} f, f\right\rangle \\
& =\left\langle\left(T_{1}^{2}-T_{1}+i d\right) f, f\right\rangle \\
& =\left\langle T_{1} f, f\right\rangle+\left\langle\left(i d-T_{1}\right)^{2} f, f\right\rangle \\
& =\left\langle T_{1} f, f\right\rangle+\left\|T_{2} f\right\|^{2},
\end{aligned}
$$

and also

$$
\left\langle T_{1} f, f\right\rangle+\left\|T_{2} f\right\|^{2} \geq\left\langle\frac{k}{(k+1)} f, f\right\rangle+\left\|T_{2} f\right\|^{2} \geq \frac{k}{(k+1)}\|f\|^{2} .
$$

Remark 3.1. Notice that for $k>3$, from the inequality $\frac{3}{4}<\frac{k}{k+1}$, it follows that inequality (3.2) is an improvement of the inequality proved in [9, Theorem 2.1].

For $k=3,4,5,6,7, \ldots$, the correspondence values of $\frac{k}{k+1}$ or $1-\frac{1}{k+1}$ are $0.75<0.8<$ $0.83<0.86<0.87<\cdots$, respectively. Hence, by increasing $k$, we see that $1-\frac{1}{k+1}$ is rapidly approaching to 1 . Therefore, inequality (3.2) is better in application and we use it for frames.

Theorem 3.2. Let $\left\{f_{i}\right\}_{i \in I}$ be a frame for the Hilbert space $\mathbb{H}$ with frame operator $S$ and canonical dual frame $\left\{\tilde{f}_{i}\right\}_{i \in I}$. For every $\emptyset \neq J \subset I$, if $S^{-\frac{1}{2}} S_{J} S^{-\frac{1}{2}} \geq \frac{k}{k+1} i d$, where $k \in \mathbb{N}$ and $k \geq 3$, then

$$
\begin{aligned}
\frac{k}{k+1} \sum_{i \in I}\left|\left\langle f, f_{i}\right\rangle\right|^{2} & \leq \sum_{i \in J}\left|\left\langle f, f_{i}\right\rangle\right|^{2}+\sum_{i \in I}\left|\left\langle S_{J^{c}} f, \tilde{f}_{i}\right\rangle\right|^{2} \\
& =\sum_{i \in J^{c}}\left|\left\langle f, f_{i}\right\rangle\right|^{2}+\sum_{i \in I}\left|\left\langle S_{J} f, \tilde{f}_{i}\right\rangle\right|^{2}, \quad \text { for } f \in \mathbb{H} .
\end{aligned}
$$

Proof. For every $J \subset I$, we have $S_{J}+S_{J^{c}}=S$, and hence $S^{-\frac{1}{2}} S_{J} S^{-\frac{1}{2}}+S^{-\frac{1}{2}} S_{J^{c}} S^{-\frac{1}{2}}=$ $i d$. By our assumptions and taking $T_{1}=S^{-\frac{1}{2}} S_{J} S^{-\frac{1}{2}}, T_{2}=S^{-\frac{1}{2}} S_{J^{c}} S^{-\frac{1}{2}}$ and $S^{\frac{1}{2}} f$ instead of $f$ in Lemma 3.1, we get

$$
\frac{k}{k+1}\left\|S^{\frac{1}{2}} f\right\|^{2} \leq\left\langle S^{-\frac{1}{2}} S_{J} f, S^{\frac{1}{2}} f\right\rangle+\left\|S^{-\frac{1}{2}} S_{J^{c}} f\right\|^{2}=\left\langle S^{-\frac{1}{2}} S_{J^{c}} f, S^{\frac{1}{2}} f\right\rangle+\left\|S^{-\frac{1}{2}} S_{J} f\right\|^{2},
$$

or equivalently,

$$
\frac{k}{k+1}\langle S f, f\rangle \leq\left\langle S_{J} f, f\right\rangle+\left\langle S^{-1} S_{J^{c}} f, S_{J^{c}} f\right\rangle=\left\langle S_{J^{c}} f, f\right\rangle+\left\langle S^{-1} S_{J} f, S_{J} f\right\rangle .
$$

Therefore,

$$
\begin{aligned}
\frac{k}{k+1} \sum_{i \in I}\left|\left\langle f, f_{i}\right\rangle\right|^{2} & \leq \sum_{i \in J}\left|\left\langle f, f_{i}\right\rangle\right|^{2}+\sum_{i \in I}\left|\left\langle S_{J^{c}} f, \tilde{f}_{i}\right\rangle\right|^{2} \\
& =\sum_{i \in J^{c}}\left|\left\langle f, f_{i}\right\rangle\right|^{2}+\sum_{i \in I}\left|\left\langle S_{J} f, \tilde{f}_{i}\right\rangle\right|^{2} .
\end{aligned}
$$

For $k=3$ inequality (3.3) is the same as the inequality proved in [9, Theorem 2.2] and for $k>3$ it is an improvement for it. 
In the following, we give an improvement for alternate dual frames. We first improve an inequality given in [9] for operators under conditions.

Lemma 3.2. If $T_{1}, T_{2} \in L(\mathbb{H})$ satisfy $T_{1}+T_{2}=i d$ and $\operatorname{Re} T_{1} \geq \frac{k}{k+1} i d$, where $k \in \mathbb{N}$ and $k \geq 3$, then

$$
\frac{k}{k+1} i d \leq T_{1}^{*} T_{1}+\frac{1}{2}\left(T_{2}^{*}+T_{2}\right)=T_{2}^{*} T_{2}+\frac{1}{2}\left(T_{1}^{*}+T_{1}\right) .
$$

Proof. From our assumptions, we have

$$
\begin{aligned}
T_{1}^{*} T_{1}+\frac{1}{2}\left(T_{2}^{*}+T_{2}\right) & =T_{1}^{*} T_{1}+\frac{1}{2}\left(i d-T_{1}^{*}+i d-T_{1}\right) \\
& =T_{1}^{*} T_{1}-\frac{1}{2}\left(T_{1}^{*}+T_{1}\right)+i d \\
& =\left(i d-T_{1}^{*}\right)\left(i d-T_{1}\right)+\frac{1}{2}\left(T_{1}^{*}+T_{1}\right) \\
& =T_{2}^{*} T_{2}+\frac{1}{2}\left(T_{1}^{*}+T_{1}\right) .
\end{aligned}
$$

And also $T_{2}^{*} T_{2}+\frac{1}{2}\left(T_{1}^{*}+T_{1}\right)=T_{2}^{*} T_{2}+\operatorname{Re} T_{1} \geq \frac{k}{k+1} i d$.

Note that, for $k=3$, inequality (3.4) is the same as the inequality proved in $[9$, Theorem 3.1] and for every $k>3$, inequality (3.4) is its improvement.

Theorem 3.3. Let $\left\{f_{i}\right\}_{i \in I}$ be a frame for the Hilbert space $\mathbb{H}$ and let $\left\{g_{i}\right\}_{i \in I}$ be an alternate dual frame of $\left\{f_{i}\right\}_{i \in I}$. For every $J \subset I$ and $f \in \mathbb{H}$, if $\operatorname{Re}\left\langle\left(\sum_{i \in J}\left\langle f, g_{i}\right\rangle f_{i}\right), f\right\rangle \geq$ $\frac{k}{k+1}\langle f, f\rangle$, where $k \in \mathbb{N}$ and $k \geq 3$, then

$$
\begin{aligned}
\frac{k}{k+1}\|f\|^{2} & \leq \operatorname{Re} \sum_{i \in J}\left\langle f, g_{i}\right\rangle \overline{\left\langle f, f_{i}\right\rangle}+\left\|\sum_{i \in J^{c}}\left\langle f, g_{i}\right\rangle f_{i}\right\|^{2} \\
& =\operatorname{Re} \sum_{i \in J^{c}}\left\langle f, g_{i}\right\rangle \overline{\left\langle f, f_{i}\right\rangle}+\left\|\sum_{i \in J}\left\langle f, g_{i}\right\rangle f_{i}\right\|^{2}, \quad \text { for } f \in \mathbb{H} .
\end{aligned}
$$

Proof. For every $J \subset I$, define the bounded linear operator $Z_{J}$ on $\mathbb{H}$ by

$$
Z_{J} f:=\sum_{i \in J}\left\langle f, g_{i}\right\rangle f_{i}
$$

By the Cauchy-Schwartz inequality, it follows that this series converges unconditionally. Since $Z_{J}+Z_{J^{c}}=i d$, by Lemma 3.1, for every $f \in \mathbb{H}$, we have

$$
\begin{aligned}
\frac{k}{k+1}\langle f, f\rangle & \leq\left\langle Z_{J}^{*} Z_{J} f, f\right\rangle+\frac{1}{2}\left\langle\left(Z_{J^{c}}^{*}+Z_{J^{c}}\right) f, f\right\rangle \\
& =\left\langle Z_{J^{c}}^{*} Z_{J^{c}} f, f\right\rangle+\frac{1}{2}\left\langle\left(Z_{J}^{*}+Z_{J}\right) f, f\right\rangle
\end{aligned}
$$


or

$$
\begin{aligned}
\frac{k}{k+1}\|f\|^{2} & \leq\left\|K_{J} f\right\|^{2}+\frac{1}{2}\left(\overline{\left\langle Z_{J^{c}} f, f\right\rangle}+\left\langle Z_{J^{c}} f, f\right\rangle\right) \\
& =\left\|Z_{J^{c}} f\right\|^{2}+\frac{1}{2}\left(\overline{\left\langle Z_{J} f, f\right\rangle}+\left\langle Z_{J} f, f\right\rangle\right) .
\end{aligned}
$$

Hence,

$$
\begin{aligned}
\frac{k}{k+1}\|f\|^{2} & \leq\left\|\sum_{i \in J}\left\langle f, g_{i}\right\rangle f_{i}\right\|^{2}+\operatorname{Re}\left\langle\sum_{i \in J^{c}}\left\langle f, g_{i}\right\rangle f_{i}, f\right\rangle \\
& =\left\|\sum_{i \in J^{c}}\left\langle f, g_{i}\right\rangle f_{i}\right\|^{2}+\operatorname{Re}\left\langle\sum_{i \in J}\left\langle f, g_{i}\right\rangle f_{i}, f\right\rangle,
\end{aligned}
$$

and the proof is completed.

Note that, for $k=3$, inequality (3.5) is the same as the inequality proved in $[9$, Theorem 3.2] and for every $k>3$, inequality (3.5) is its improvement. Finally, we give a more general result.

Theorem 3.4. Let $\left\{f_{i}\right\}_{i \in I}$ be a frame for the Hilbert space $\mathbb{H}$ and let $\left\{g_{i}\right\}_{i \in I}$ be an alternate dual frame of $\left\{f_{i}\right\}_{i \in I}$. For every $f \in \mathbb{H}$, if $\operatorname{Re}\left\langle\left(\sum_{i \in J}\left\langle f, g_{i}\right\rangle f_{i}\right), f\right\rangle \geq \frac{k}{k+1}\langle f, f\rangle$, where $k \in \mathbb{N}$ and $k \geq 3$, then for any bounded sequence $\left\{w_{i}\right\}_{i \in I}$, we have

$$
\begin{aligned}
\frac{k}{k+1}\|f\|^{2} & \leq \operatorname{Re} \sum_{i \in I} w_{i}\left\langle f, g_{i}\right\rangle \overline{\left\langle f, f_{i}\right\rangle}+\left\|\sum_{i \in I}\left(1-w_{i}\right)\left\langle f, g_{i}\right\rangle f_{i}\right\|^{2} \\
& =\operatorname{Re} \sum_{i \in I}\left(1-w_{i}\right)\left\langle f, g_{i}\right\rangle \overline{\left\langle f, f_{i}\right\rangle}+\left\|\sum_{i \in I} w_{i}\left\langle f, g_{i}\right\rangle f_{i}\right\|^{2}, \quad \text { for } f \in \mathbb{H} .
\end{aligned}
$$

Proof. In Lemma 3.2, we put

$$
T_{1} f=\sum_{i \in I} w_{i}\left\langle f, g_{i}\right\rangle f_{i}, \quad T_{2} f=\sum_{i \in I}\left(1-w_{i}\right)\left\langle f, g_{i}\right\rangle f_{i} .
$$

The result is obtained from Theorem 3.3, if we take $J \subset I$ and

$$
w_{i}= \begin{cases}1, & \text { for } i \in J \\ 0, & \text { for } i \in J^{c}\end{cases}
$$

\section{REFERENCES}

[1] R. Balan, P. G. Casazza, D. Edidin and G. Kutyniok, A new identity for Parseval frames, Proc. Amer. Math. Soc. 135 (2007), 1007-1015.

[2] P. G. Casazza, The art of frame theory, Taiwanese J. Math. 4 (2000), 129-201.

[3] P. G. Casazza and G. Kutyniok, Frames of subspaces, in: Wavelets, Frames and Operator theory, Contemp. Math. 345, Amer. Math. Soc., Providence, RI, 2004, 87-113,.

[4] O. Christensen, An Introduction to Frames and Riesz Bases, Birkhauser/Springer, Basel, 2016.

[5] I. Daubechies, Ten Lectures on Wavelets, SIAM, Philadelphia, PA, 1992.

[6] J. Duffin and A. C. Schaeffer, A class of nonharmonic Fiurier series, Trans. Amer. Math. Soc. 72 (1952), 341-366. 
[7] Y. C. Eldar and G. D. Forney, Optimal tight frames and quantum measurement, IEEE Trans. Inform. Theory 48 (2002), 599-610.

[8] Sh. Furuichi, Refined Young inequalities with Specht's ratio, J. Egyptian Math. Soc. 20 (2012), 46-49.

[9] P. Gavruta, On some identities and inequalities for frames in Hilbert spaces, J. Math. Anal. Appl. 321 (2006), 469-478.

[10] L. Gavruta, Frames for operators, Appl. Comput. Harmon. Anal. 32 (2012), 139-144.

[11] Q. P. Guo, J. S. Leng and H. B. Li, Some equalities and inequalities for fusion frames, Springer Plus 5 (2016), Article ID 121, 10 pages.

[12] D. Han and D. R. Larson, Frames, bases and group representations, Mem. Amer. Math. Soc. 147 (2000), 94 pages.

[13] R. Vale and S. Waldron, Tight frames and their symmetries, Constr. Approx. 21 (2005), 83-112.

\author{
${ }^{1}$ Department of Mathematics, \\ Mashhad Branch, Islamic Azad University, \\ MASHHAD, IRAN \\ Email address: fsultanzadeh@gmail.com \\ Email address: mhassanimath@gmail.com \\ Email address: math.erfanian@gmail.com \\ ${ }^{2}$ Department of Pure Mathematics, \\ FERDOWSI UNIVERSITY OF MASHHAD, \\ MASHHAD, IRAN \\ Email address: kamyabi@um.ac.ir
}

